\title{
A NEW MEMS-BASED QUARTZ RESONATOR TECHNOLOGY
}

\author{
David T. Chang, Frederic P. Stratton, Deborah J. Kirby, \\ Richard J. Joyce, Tsung-Yuan Hsu, Randall L. Kubena \\ Sensors and Materials Laboratory, HRL Laboratories, LLC \\ Malibu, CA 90265-4799
}

\begin{abstract}
We report on the development of a new quartz resonator technology that allows for the processing and integration of VHF to UHF high-Q oscillators and filters with high-speed silicon or III-V electronics. This paper describes the successful demonstration of new wafer bonding and dry plasma etching processes that make quartz-MEMS technology possible. In addition, we present impedance, Q, and temperature sensitivity data along with comparison to 3-D harmonic and thermal analysis of VHF-UHF resonators.
\end{abstract}

\section{INTRODUCTION}

Modern communication systems such as programmable radios and GPS receivers require ultra small, high frequency filters or oscillators with extremely good temporal and thermal stabilities, high resonant quality-factors, and excellent RF matching characteristics. Discrete bulk acoustic wave devices such as quartz resonators have been the prevailing choice for such applications because single crystal quartz has several attractive material properties. It is a low loss (high Q) piezoelectric material with zero temperature coefficient and stress compensated crystal cuts. In addition, its chemically inert surface makes quartz a candidate for stable frequency operations. However, current manufacturing technology for quartz resonators does not provide a straightforward method for reducing the size and thereby increasing the frequency of operation into the UHF range [1]. Furthermore, integrating large arrays of precisely tuned structures with high-frequency RF electronics and vacuum packaging the resulting chip at wafer level are not possible with present techniques. Polysilicon surface micromachining technology has enabled the creation of on-chip UHF resonators with high Q values [2]. However, these devices suffer from extremely large motional resistance $(>1 \mathrm{k} \Omega)$, making them less desirable for low impedance $(\sim 50 \Omega)$ RF applications.

Recent advancements in microfabrication, especially in the areas of precision wafer bonding and plasma etching, have enabled us to fabricate miniaturized quartz on-chip resonators working in the VHF-UHF frequency range. These resonators retain the excellent properties of discrete quartz devices while providing a low-cost path for on-chip integration with electronics and waferscale packaging.

\section{DEVICE DESIGN AND SIMULATION}

In quartz resonator design, it is important to optimize energy trapping and motional resistance and to exclude spurious oscillation modes by adjusting electrode and quartz plate dimensions. We worked with Coventor to establish the first commercial piezoelectric 3-D finite element modal and impedance analysis software to design thickness-shear mode (TSM) quartz resonators and predict their frequency characteristics.
Figure 1 shows the finite element simulation result for an ATcut TSM quartz resonator of dimensions $265 \mu \mathrm{m}(\mathrm{L})$ x $80 \mu \mathrm{m}(\mathrm{W})$ $\mathrm{x} 10 \mu \mathrm{m}(\mathrm{T})$ with top and bottom gold (Au) electrodes of dimensions, $200 \mu \mathrm{m}(\mathrm{L}) \times 50 \mu \mathrm{m}(\mathrm{W}) \times 0.12 \mu \mathrm{m}(\mathrm{T})$. The 3-D modal analysis indicates that at $149 \mathrm{MHz}$ this design has excellent energy confinement under the electrodes and pure thickness-shear oscillation without any anharmonic modes near this resonance.

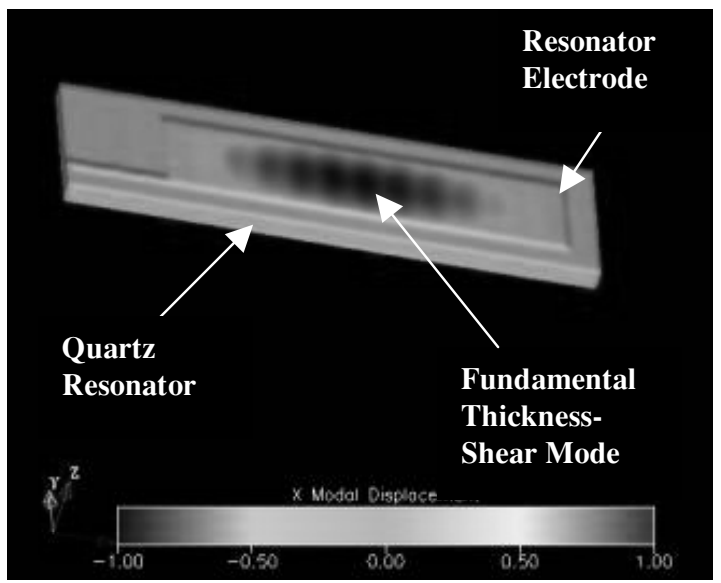

Figure 1. 3-D modal pattern of a $149 \mathrm{MHz}$ thickness-shear quartz resonator.

Impedance analysis of this resonator design provides the frequency-dependent I-V characteristics of the device. Using the admittance routines available from the Coventor software package, we calculated the admittance versus frequency plots for several of the designs on our current mask set. Figure 2 shows the admittance vs. frequency plot for the aforementioned $149 \mathrm{MHz}$ resonator design.

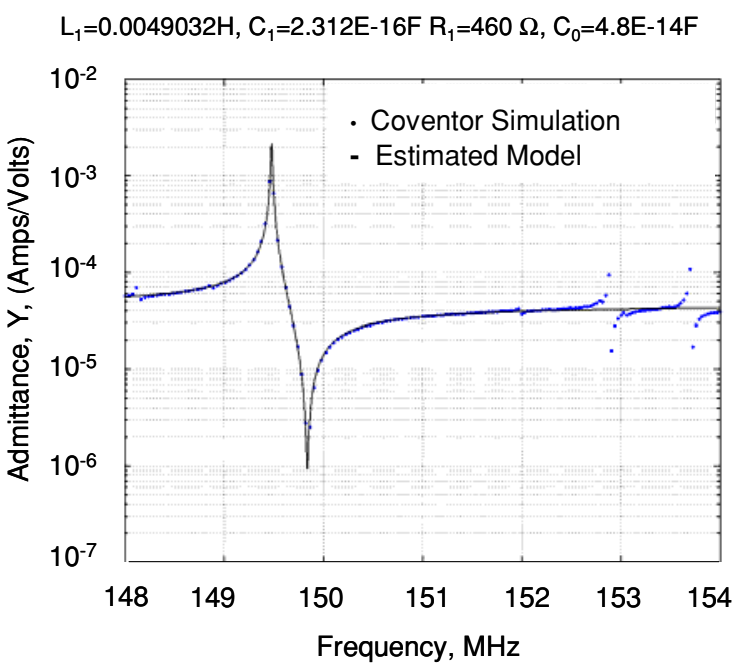

Figure 2. Simulated admittance vs. frequency plot of a $149 \mathrm{MHz}$ thickness-shear quartz resonator. 
The simulation results were then used to extract the equivalent circuit parameters for a Van Dyke circuit, and the results compare favorably with analytical data. For an assumed Q of 10,000, a 265 x 80 micron design yielded a motional resistance $R_{1}$ of $460 \Omega$, motional inductance $\mathrm{L}_{1}=4.9 \mathrm{mH}$, motional capacitance $\mathrm{C}_{1}=0.23 \mathrm{fF}$ and a static capacitance of $\mathrm{C}_{0}=48 \mathrm{fF}$.

\section{FABRICATION}

The quartz resonator fabrication process is illustrated in figure 3. The starting materials for this process are: 1) a 300- $\mu \mathrm{m}-$ thick, double-side polished AT-cut single crystal quartz blank, 2) a 500- $\mu$ m-thick, <100>-oriented, n-type, 1-10 ohm-cm, silicon handle wafer, and 3) a 300- $\mu$ m-thick host substrate such as a GaAs or a silicon wafer. The process begins with a plasma cavity etch into the silicon handle wafer $(\sim 2$ microns depth) to later accommodate the top metal electrodes of the quartz wafer. The quartz wafer is metallized with electrodes $(200 \AA \mathrm{Cr} / 1000 \AA \mathrm{Au})$ and then aligned and directly bonded to the silicon handle wafer using plasma-assisted room temperature bonding with EV Group's EV-620 aligner and EV-501 bonder. The bonded quartz is subsequently thinned to a thickness of approximately $10 \mu \mathrm{m}$ using conventional lapping and polishing techniques. Its thickness is further reduced to less than $10 \mu \mathrm{m}$ using an $\mathrm{SF}_{6}$-based plasma etch in a Unaxis inductively-coupled, high-density plasma etcher. Veeco surface profilometer measurements of the final quartz surface after thinning give a surface roughness of $2 \mathrm{~nm}$.

\section{Starting Materials}

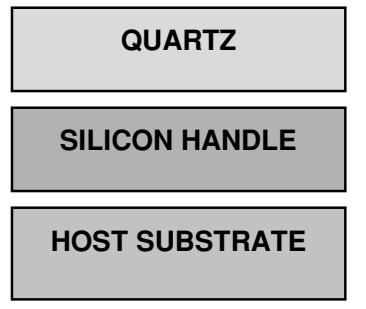

2. Etch Cavities in $\mathrm{Si}$

SILICON HANDLE

\section{Pattern and Metallize Top Electrodes on Quartz}

\section{Bond Quartz to Si Handle}
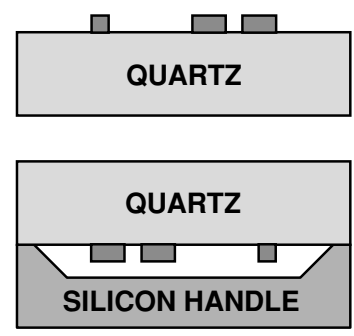

\section{Thin Quartz to $<10 \mu \mathrm{m}$}

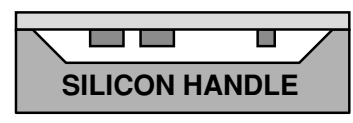

\section{Pattern and Metallize Via Holes in Quartz}

\section{Pattern and Metallize Bottom Electrodes on Quartz}

\section{Pattern and Etch Quartz Resonator}

\section{Etch Protrusion in Host Substrate}

10. Pattern and Metallize Bond Pads

\section{Bond Si/Quartz to Host Substrate}

\section{Remove Si Handle}

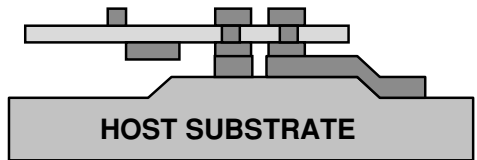

Figure 3. Fabrication process flow for the quartz resonators.

A deep reactive ion etching (DRIE) process with $\mathrm{CF}_{4}$ chemistry and bottom-side metallization $(200 \AA \mathrm{Cr} / 1000 \AA \mathrm{Au})$ create the through-wafer vias and metal interconnects to bridge the top-side metallization to bottom-side bonding pads. The continuous sheet of quartz is then patterned and etched using a thick Shipley SJR-5740 photoresist mask and a second DRIE step to delineate the resonator patterns. (Fig. 4) The detailed description of the DRIE process is presented in an earlier publication [3].

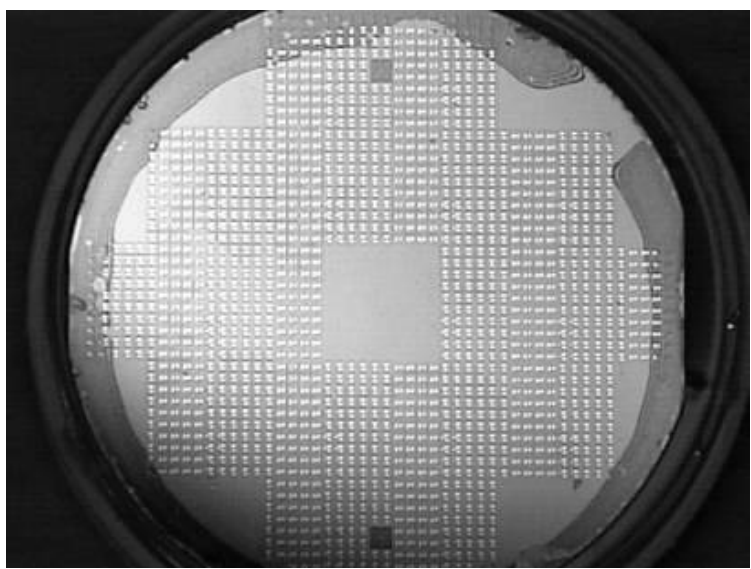

Figure 4. Quartz resonator patterns on silicon handle wafer after process step 8. 
For the host substrate, it is first patterned and etched to create $5-\mu \mathrm{m}$-tall protrusions on its surface. Then, metals are deposited on the protrusions to form the bond pads $(200 \AA \mathrm{Cr} / 5000 \AA \mathrm{Au})$ for the subsequent thermal compression bond. In this bonding step, the $\mathrm{Si}$ /quartz pair is first aligned to the host substrate using $\mathrm{EV}$ Group's EV-620 aligner and then bonded at $300^{\circ} \mathrm{C}$ in the EV-501 wafer bonder using a compression pressure of $10 \mathrm{MPa}$. Finally, the silicon handle wafer is removed using either an $\mathrm{SF}_{6}$ plasma etch or a wet TMAH process to leave the individual quartz resonators on the host substrate. Completed resonators are shown in both the microscope photograph (Fig. 5) and an SEM image. (Fig. 6)

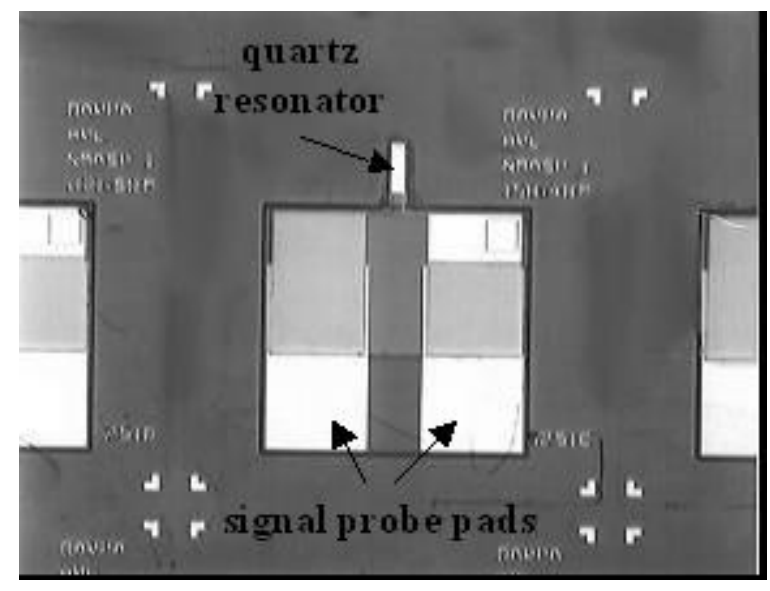

Figure 5. Microscope photograph of a completed resonator.

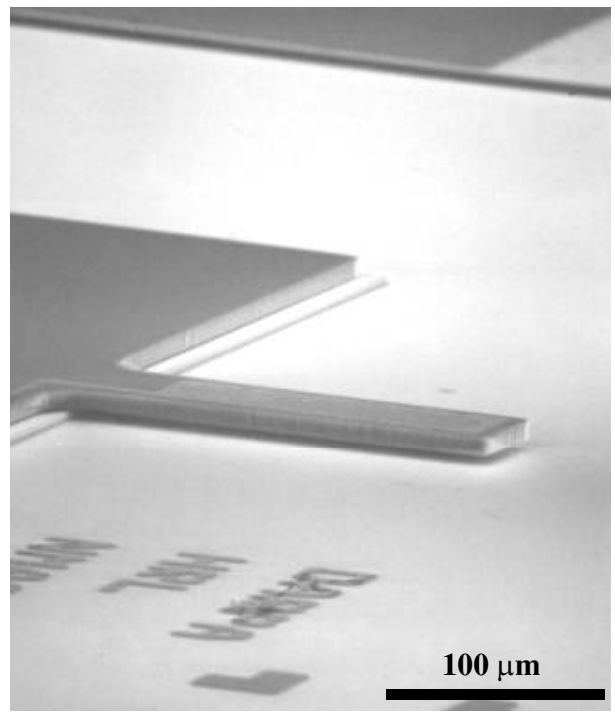

Figure 6. SEM photograph of a completed resonator.

This fabrication process provides a path for integrating large resonator arrays with high frequency RF electronics since the lowtemperature process is compatible with standard silicon and III-V compound semiconductor processes. It also allows conventional packaging techniques such as anodic, glass frit, and solder bonding to hermetically seal the resonators at the wafer level because the permanent $\mathrm{Au}-\mathrm{Au}$ thermal compression bond for the resonators can withstand a post-processing temperature of at least $400^{\circ} \mathrm{C}$, which is ample for the aforementioned sealing processes.

\section{EXPERIMENTAL DETAILS}

Several different designs of AT-cut shear-mode resonators have been tested using Cascade microprobes and an Agilent 4991A impedance analyzer at room temperature. One of the keys to making measurements on ultra-small piezoelectric resonators is compensating for stray capacitance during wafer-level probing. Capacitance compensation below $10^{-13} \mathrm{~F}$ is generally required. For a 1.49- $\mu \mathrm{m}$-thick quartz resonator with electrode dimensions of 160 $\mu \mathrm{m} \times 50 \mu \mathrm{m} \times 0.1 \mu \mathrm{m}$, we obtained a fundamental frequency of $591 \mathrm{MHz}$ and a $\mathrm{Q}$ value of 13,470 in air for this device (Fig. 7). This yields an $\mathrm{f} \times \mathrm{Q}$ product of $8 \times 10^{12} \mathrm{~Hz}$, which is within a factor of two of the highest frequency-Q product reported to date for any quartz resonator [4]. The motional resistance of this resonator was measured to be $40 \Omega$, thus providing good impedance matching to external transmission lines. These results indicate that we have obtained good energy confinement and very low damage of the quartz during processing. In addition, temperature test data showed that these devices have a linear temperature coefficient of frequency of about $-5 \mathrm{ppm} /{ }^{\circ} \mathrm{C}$ near room temperature (RT), which is within a factor of two of the best reported temperature coefficient to date for an on-chip resonator without external compensation [5]. Thermal analysis of our designs and quartz cutangles predict a linear frequency shift near room temperature of -4 $\mathrm{ppm} /{ }^{\circ} \mathrm{C}$. Previous data for AT-cut resonators indicate that a maximum frequency deviation of $50 \mathrm{ppm}$ from $-40^{\circ} \mathrm{C}$ to $85^{\circ} \mathrm{C}$ (near zero TC at RT) is possible with an optimized quartz cut [6].

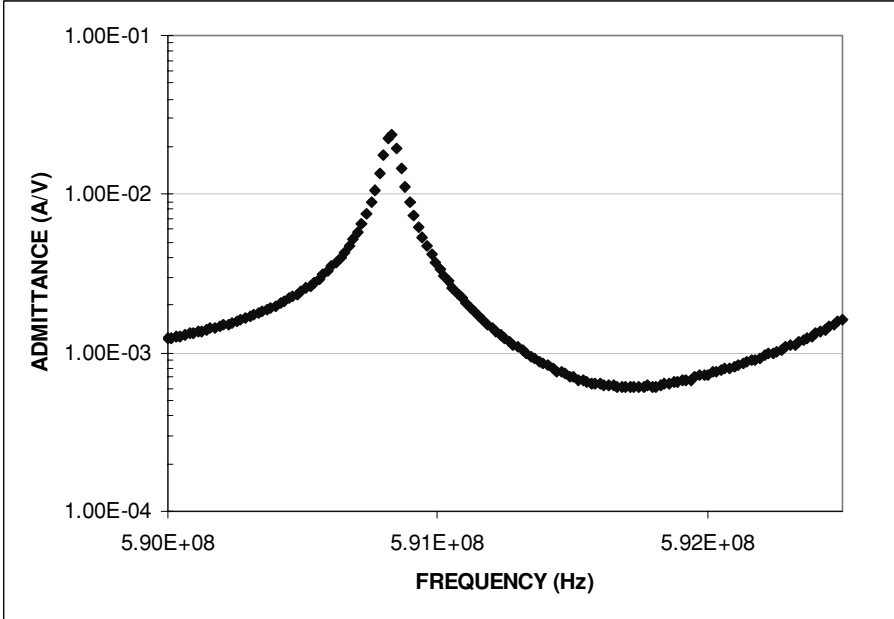

Figure 7. Measured admittance vs. frequency for a 1.49- $\mu$ m-thick, AT-cut shear-mode resonator with electrode dimensions of $160 \mu \mathrm{m}$ $x 50 \mu \mathrm{m} \times 0.1 \mu \mathrm{m}$.

\section{FREQUENCY TUNING OF RESONATORS}

For precision frequency adjustments after fabrication, we have demonstrated an ultra clean and high-resolution method of real-time mechanical tuning using focused-ion-beam (FIB) micromachining. A beam of gallium ions is focused and scanned over the surface of the top Au resonator electrode. The interaction of the ion beam with the electrode results in a controlled ejection of $\mathrm{Au}$ atoms from the resonator with sub-monolayer precision. The reduction in electrode mass leads to an increase in device 
frequency. Figure 8 depicts the modified FEI focused-ion-beam system used for frequency tuning of our quartz resonators.

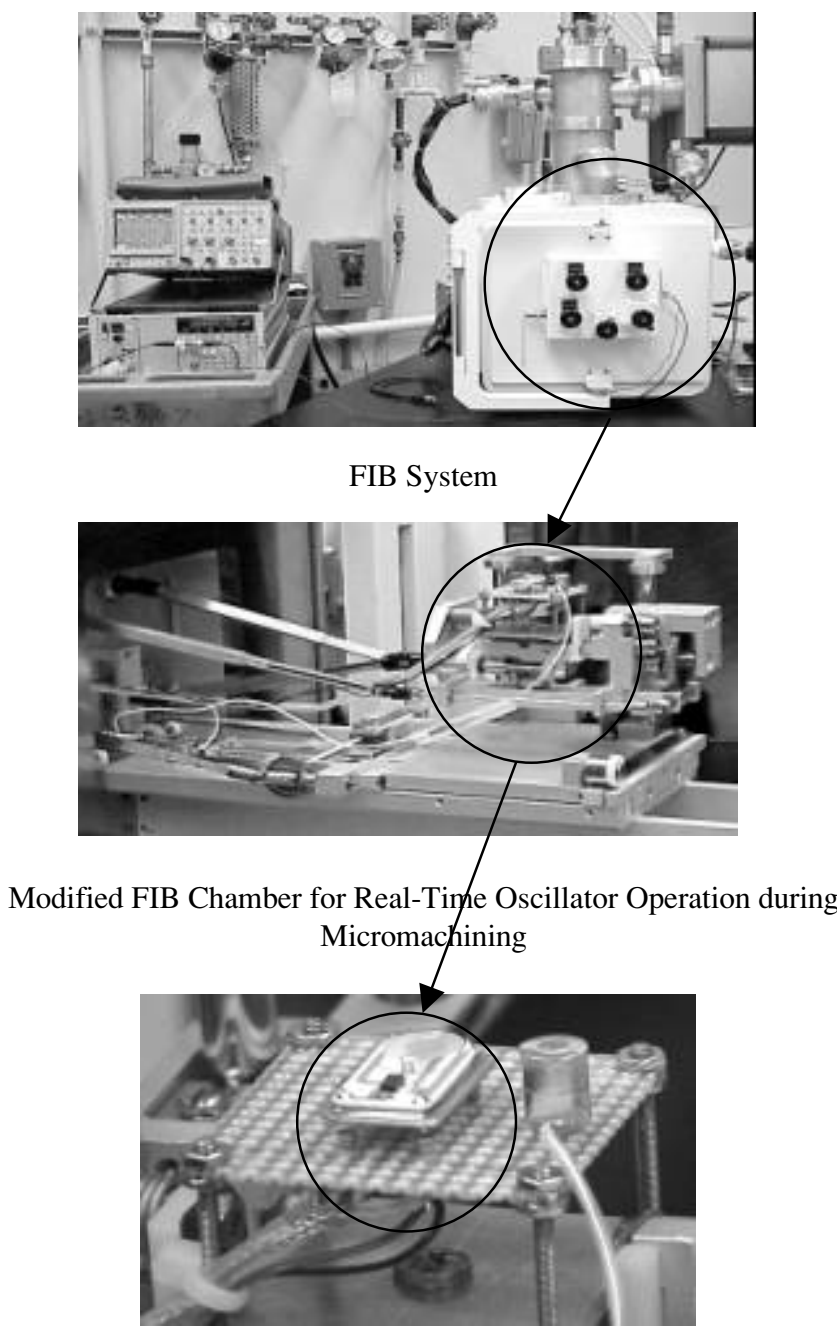

$20 \mathrm{MHz}$ Quartz Oscillator

Figure 8. Focused-ion-beam (FIB) system for frequency tuning of quartz resonators.

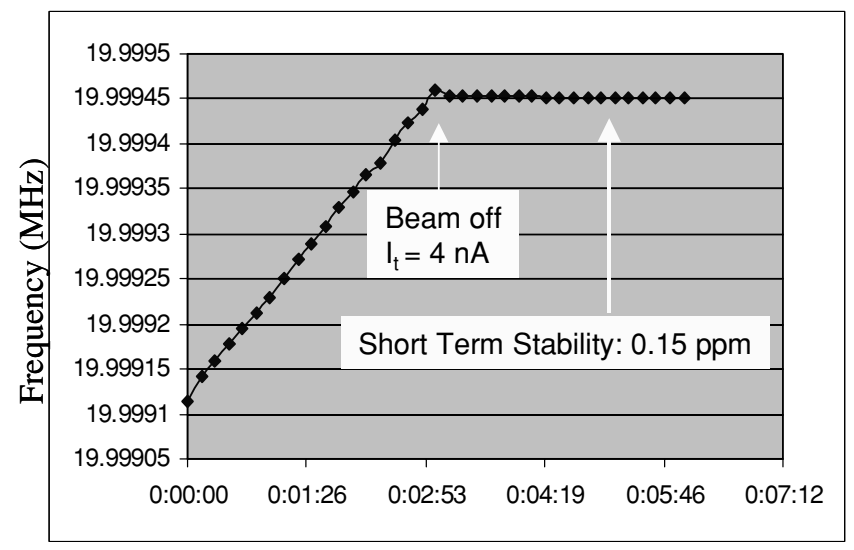

Milling Time (hr./min./sec.)

Figure 9. FIB tuning of an AT-cut quartz resonator. The initial resonant frequency of the resonator was $19.999113 \mathrm{MHz}$. After the ion beam is turned off, the resonant frequency became 19.999454 $\mathrm{MHz}$. The frequency eventually stabilized to $19.999451 \mathrm{MHz}$ to represent a short- term stability of $150 \mathrm{ppb}$.
We achieved a frequency modification of $338 \mathrm{~Hz}$ on a 20 $\mathrm{MHz}$ AT-cut quartz resonator after 3 minutes of milling under a beam current of $4 \mathrm{nA}$ and a voltage of $25 \mathrm{kV}$. (Fig. 9) After removal from the FIB vacuum chamber and a re-pump down to vacuum $\left(10^{-5}\right.$ Torr $)$, the frequency stability was observed to be roughly $1 \mathrm{~Hz}$ for this $20 \mathrm{MHz}$ quartz resonator. For ultra-small UHF resonators, FIB tuning is ideal due to its high spatial resolution and monolayer milling control.

\section{CONCLUSIONS}

We have developed a new VHF-UHF quartz resonator technology which can be integrated with RF electronics and is compatible with MEMS-based wafer-scale vacuum packaging. For a quartz thickness-shear mode resonator thinned to $1.49 \mu \mathrm{m}$, we measured a resonant frequency of $591 \mathrm{MHz}$ and a $\mathrm{Q}$ of 13,470 in air. This device has an excellent motional resistance of $40 \Omega$ and a temperature coefficient of $-5 \mathrm{ppm} /{ }^{\circ} \mathrm{C}$. We have also demonstrated that FIB micromachining can be used for precise frequency tuning of resonator with $150 \mathrm{ppb}$ accuracy. Future work will emphasize higher frequency performance using thinner aluminum electrodes and optimizing the temperature stability.

\section{ACKNOWLEDGMENTS}

The authors would like to thank Dr. John Vig of US Army CECOM and Prof. Yook-Kong Yong of Rutgers University for their valuable technical discussions and support. We also acknowledge the modeling support of Matt Lim and Prof. Robert M'Closkey from UCLA. This project is being funded by DARPA's Microsystems Technology Office under contract DAAB07-02-C-P613.

\section{REFERENCES}

1. Statek Corporation, Technical Note 28, Rev. A, "An UltraMiniature Low-Profile AT Quartz Resonator."

2. C. T.-C. Nguyen, "Frequency-Selective MEMS for Miniaturized Low-Power Communication Devices," IEEE Transactions on Microwave Theory and Techniques, vol. 47, no 8, pp 1486-1503, 1999.

3. D. Chang, F. Stratton, R. Kubena, R. Joyce, "Optimized DRIE Etching of Ultra-Small Quartz Resonators," Proceedings of the 2003 IEEE International Frequency Control Symposium, pp 829$832,2003$.

4. R. Smythe and R. Angove, "Chemically-Milled UHF SC-Cut Resonators," Proceedings of the 1988 IEEE International Frequency Control Symposium, pp 73-77, 1988.

5. W.-T. Hsu, J. R. Clark, C. T.-C. Nguyen, "A Sub-Micron Capacitive Gap Process for Multiple-Metal-Electrode Lateral Micromechanical Resonator," Proceedings of the $14^{\text {th }}$ IEEE International Conference on Micro Electro Mechanical Systems, pp. $349-352,2001$.

6. Microchip Technology, Inc. Technical Note AN 588. 